\title{
Shock wave focusing using geometrical shock dynamics
}

\author{
J. E. Cates and B. Sturtevant \\ Graduate Aeronautical Laboratories, California Institute of Technology, Pasadena, California 91125
}

(Received 7 January 1997; accepted 23 April 1997)

\begin{abstract}
A finite-difference numerical method for geometrical shock dynamics has been developed based on the analogy between the nonlinear ray equations and the supersonic potential equation. The method has proven to be an efficient and inexpensive tool for approximately analyzing the focusing of weak shock waves, where complex nonlinear wave interactions occur over a large range of physical scales. The numerical results exhibit the qualitative behavior of strong, moderate, and weak shock focusing observed experimentally. The physical mechanisms that are influenced by aperture angle and shock strength are properly represented by geometrical shock dynamics. Comparison with experimental measurements of the location at which maximum shock pressure occurs shows good agreement, but the maximum pressure at focus is overestimated by about $60 \%$. This error, though large, is acceptable when the speed and low cost of the method is taken into consideration. The error is primarily due to the under prediction of disturbance speed on weak shock fronts. Adequate resolution of the focal region proves to be particularly important to properly judge the validity of shock dynamics theory, under-resolution leading to overly optimistic conclusions. (C) 1997 American Institute of Physics. [S1070-6631(97)02109-0]
\end{abstract}

\section{INTRODUCTION}

The focusing of shock waves produces localized high pressures in the focal region, and the shock emerges from the focus with the front geometry fundamentally changed. Understanding the mechanisms of focusing is critical because converging fronts occur frequently, for example when passing through nonuniform media or reflecting from curved surfaces. The high pressures localized near the focus may be beneficial, as in shock wave lithotripsy, or detrimental, as in superbooms from supersonic aircraft. The change in shock geometry downstream of the focus has significant implications for shock stability, sonic boom propagation, and sonoluminescence.

One recent application of shock wave focusing is extracorporeal shock wave lithotripsy (ESWL). In this treatment for kidney stone disease, weak converging shock waves are generated in water outside the patient's body and shaped to focus on the stone. In the focal region, the shock pressure increases to about $20 \mathrm{MPa}$, sufficiently strong to fragment the stone, although the mechanism of fracture is not completely understood. Over the course of several thousand shocks, the stone is shattered into pieces small enough to be eliminated naturally. To better understand the source of tissue injury and the primary mechanism of stone fragmentation in ESWL, understanding of the amplitude and geometry of the wave field in the focal region is required.

The experiments of Sturtevant and Kulkarny ${ }^{1}$ first demonstrated the complex behavior at the focus. They reflected planar shocks from a parabolic reflector to a line focus. The three types of focusing behavior observed are shown in Fig. 1 , reproduced from Kulkarny. ${ }^{2}$ Solid lines represent the shock fronts after reflection from the parabola shown at the left. For sound pulses in acoustic theory, the rays continue to a perfect focus; afterward the shock front is crossed and folded as shown in Fig. 1(a). At the focus in acoustic theory, the ray tube area goes to zero and the shock amplitude is infinite. For weak shocks, Fig. 1(b), a Mach stem is formed near the focus. The triple point initially moves outward, but is eventually pushed back to the centerline, and the shock front becomes crossed and folded. For strong shocks, Fig. 1(d), a Mach stem is again formed, but the triple point moves continuously outward as the front advances past the acoustic focus. In this case after focus the front is not crossed or folded. A transition case for moderate strength shocks is also observed, Fig. 1(c), where the triple point initially moves outward from the centerline. Further downstream, the triple point is swept back toward the centerline but never reaches the centerline. Instead the triple point begins to move outward again. Later experiments with weak shocks have observed similar behavior. ${ }^{3-5}$

The shock strength near the focus of a shock wave lithotriptor, $M \sim 1.03-1.05$, corresponds to the weak shock case, Fig. 1(b). For these weaker shocks, the behavior near the focus approaches the prediction of acoustics. The focal amplification is higher, and the focal region is narrower and located closer to the acoustic focus than for stronger shocks. ${ }^{1,3}$ For smaller aperture angles, corresponding to shallow reflectors, at equal initial Mach numbers, the focal pressure is lower and the focus is located farther upstream of the acoustic focus than for larger aperture angles. For weak shocks with large aperture angles, the location of the maximum pressure can lie beyond the acoustic focus. For stronger shocks or smaller aperture angles, the focus is located upstream of the acoustic focus. ${ }^{1,5}$

For the purpose of modeling this behavior numerically, Whitham's theory of geometrical shock dynamics ${ }^{6,7}$ offers an appealing alternative to a full Euler solution. By describing only the motion and geometry of the wavefronts, the dimensionality of the problem is reduced by one and the complexity of numerical calculations is greatly reduced. The geometry of the wave field, the most important viewpoint for understanding the shock wave physics of complex wave interactions, is emphasized. 


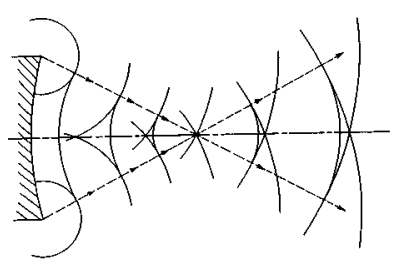

(a) Sound pulse (acoustic theory)

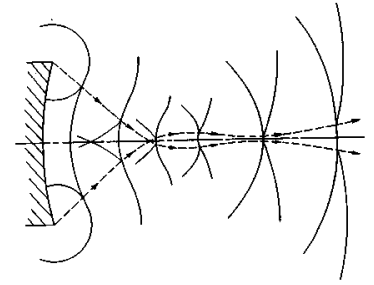

(c) Moderate strength

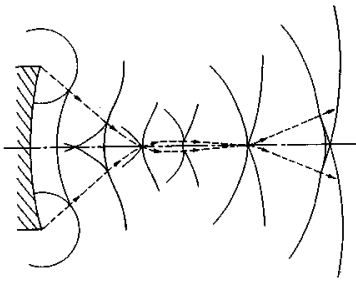

(b) Weak shock

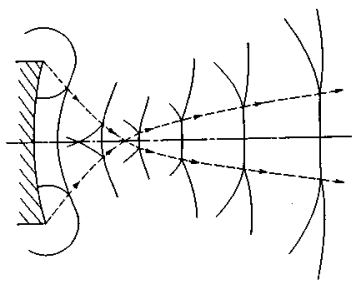

(d) Strong shock
FIG. 1. Types of focusing behavior observed in experiment, reproduced from Kulkarny.

In geometrical shock dynamics, the shock propagates down ray tubes which are normal to the front, as in geometrical acoustics. However, shock dynamics is nonlinear, in that the velocity of the shock depends on its strength. To close the system, an area-Mach number relation is introduced to relate the shock strength, represented by the Mach number, to the variation of the ray tube area. To accomplish this, the propagation of the shock down the ray tube is treated as propagation down a tube with solid walls and a slowly varying cross-sectional area. For this problem, by ignoring the effect of disturbances overtaking the shock from behind, a relationship can be derived for the Mach number as a function of area. Since the theory ignores the interaction of the shock with the flow behind it, it is expected that the theory should be particularly appropriate for problems with accelerating shocks and/or dominated by geometry. The accuracy of the approximation for specific problems is difficult to assess in advance, but geometrical shock dynamics has proven to be accurate for a wide range of problems. In problems where a comparison with full compressible flow solutions is available, shock dynamics has shown good agreement for strong shocks, and is generally less reliable for weak shocks. Various efforts to extend shock dynamics has been proposed, but none have gained wide acceptance.

Henshaw et al., ${ }^{8}$ using a front-tracking method to solve the equations of shock dynamics, analyzed shock focusing and compared calculated results with the experiments of Sturtevant and Kulkarny. ${ }^{1}$ They showed that shock dynamics qualitatively reproduces the weak-, moderate- and strongshock behavior illustrated in Fig. 1, and that, by adjusting the flatness of the initial shock front, they could quantitatively match the maximum strength at the focus of a shock wave initially of strength $M_{0}=1.1$.

Another approximate method which has been used for calculating shock and ultrasonic wave focusing solves the 2D Burgers equation (also known as transonic small disturbance theory, the nonlinear parabolic wave equation and the
KZK equation ${ }^{9,10}$ ). This approximation yields the entire pressure field. For problems in acoustics a formulation treating the signaling problem has been utilized, in which time series of pressure are specified on a source plane. To date, shock tracking or capturing algorithms or nonuniform grids have not been used in solving the KZK equation to resolve shock waves of strengths typically found in lithotriptors. No comparisons with experiments such as those of Sturtevant and Kulkarny ${ }^{1}$ are available. Brio and Hunter ${ }^{11}$ showed that the inviscid form of the 2D Burgers equation (the $\mathrm{ZK}$ equation) does not support triple shock intersections, a crucial feature of shock focusing [Figs. 1(b)-(d)]. Rather, it yields so-called von Neumann reflection, in which the reflected wave is diffuse. They also showed that an under-resolved numerical calculation of the ZK equation erroneously does show triple shock intersection.

The objective of this paper is to test the validity of shock dynamics solutions of weak shock wave focusing. Owing to the large ratio of the scale of the initial curvature of the wavefront to the size of the focal spot for weak shocks, shock focusing is one of the most severe tests of numerical algorithms for wave interactions. We compare the results of the calculations with experimental results, ${ }^{1}$ and we report results for shock strengths relevant to ESWL, as low as $M_{0}=1.01$. It turns out that for these problems the fact that shock dynamics does not describe regular reflection from walls, but only Mach reflection, and also underestimates the speed of transverse waves on weak shock fronts, causes significant quantitative disagreement with experiment.

\section{NUMERICAL METHOD}

Only a small, relatively simple set of problems of interest can be worked analytically using the method of shock dynamics. Two numerical approaches have been used for a wide range of problems: the method of characteristics ${ }^{12,13}$ and front-tracking methods. ${ }^{8}$ Characteristics methods are typically more cumbersome than finite-difference methods and difficult to extend to three dimensional problems, and thus their numerical application has been fairly limited. In front-tracking methods points along the shock front are advanced along rays normal to the front according to the shock Mach number. The ray-tube area is then used to compute the Mach number along the front at the new position. The extension of front-tracking methods to three-dimensions is straightforward. However, the method requires frequent splining of the points and the addition of points in areas where the front expands and the removal of points where the front contracts.

As noted by Whitham ${ }^{7}$ the equations of geometrical shock dynamics are analogous to the supersonic potential equation, and numerical schemes for the supersonic potential equation can be adapted to shock dynamics. ${ }^{14}$ The use of finite-difference schemes offers advantages over previous characteristic and front methods. Finite-difference schemes provide conservative formulations where the effects of artificial viscosity can be clearly controlled. The method is easily extended to three dimensional problems with optimal grids for specific problems. In addition, finite-difference 
schemes are typically easier to set up and apply to specific problems than previous methods, especially for problems with weak shocks.

The equations of geometrical shock dynamics ${ }^{6,7}$ are

$$
\boldsymbol{\nabla} \cdot\left(\frac{M}{A(M)} \boldsymbol{\nabla} \alpha\right)=0, \quad M=\frac{1}{|\boldsymbol{\nabla} \alpha|} .
$$

$\alpha(\mathbf{x})=a_{0} t$ gives the shock position at time $t$, where $a_{0}$ is the undisturbed sound speed. $A(M)$ is the area-Mach number relation. For a perfect gas, the area-Mach number relation is given by

$$
A(M)=\exp \left[-\int_{M_{0}}^{M} \frac{M \lambda(M)}{M^{2}-1} d M\right],
$$

where

$$
\begin{aligned}
& \lambda(M)=\left(1+\frac{2}{\gamma+1} \frac{1-\mu^{2}}{\mu}\right)\left(1+2 \mu+\frac{1}{M^{2}}\right), \\
& \mu^{2}=\frac{(\gamma-1) M^{2}+2}{2 \gamma M^{2}-(\gamma-1)},
\end{aligned}
$$

and $\gamma$ is the ratio of specific heats. An analytical expression for the area-Mach number integral was originally given by Bryson and Gross. ${ }^{12}$ Several misprints were later pointed out by Henderson. ${ }^{15}$

For shock waves in water, such as occur in lithotripsy, the modified Tait equation of state originally proposed by Kirkwood and Bethe ${ }^{16}$ provides a convenient form for analysis. A common form of the equation which we use here assumes that the reference quantities $B$ and $\rho_{0}$, actually weak functions of entropy, are constant,

$$
p(\rho)=B\left[\left(\frac{\rho(p, T)}{\rho_{0}}\right)^{\Gamma}-1\right] .
$$

At $T=20 \mathrm{C}$, the values $\Gamma=7.15$ and $\rho_{0}=998.232 \mathrm{~kg} / \mathrm{m}^{3}$ are often used to represent water. $B$ follows from $\Gamma$ and the sound speed through the relation

$$
a^{2}=\left(\frac{\partial p}{\partial \rho}\right)_{s}=\frac{\Gamma(p+B)}{\rho},
$$

$B=303.975 \mathrm{MPa}$ for water. Using the Tait equation in the Rankine-Hugoniot conditions for mass and momentum yields a transcendental equation for the density ratio $R=\rho_{2} / \rho_{1}$ across a shock wave,

$$
R^{\Gamma+1}-\left(1+\Gamma M^{2}\right) R+\Gamma M^{2}=0 .
$$

The "shifted" pressure ratio $Z=\left(p_{2}+B\right) /\left(p_{1}+B\right)$ is given by $Z=R^{\Gamma}$. The $A(M)$ relation for the Tait equation is derived in the usual way from the characteristic relation

$$
\frac{d p}{d x}+\rho a \frac{d u}{d x}+\frac{\rho a^{2} u}{u+a} \frac{1}{A} \frac{d A}{d x}=0,
$$

by eliminating the differential of pressure using Eq. (2.6), the differential of velocity using the shock relation for mass conservation and the resulting differentials of density using Eq. (2.7), all in favor of $d M$. The result is

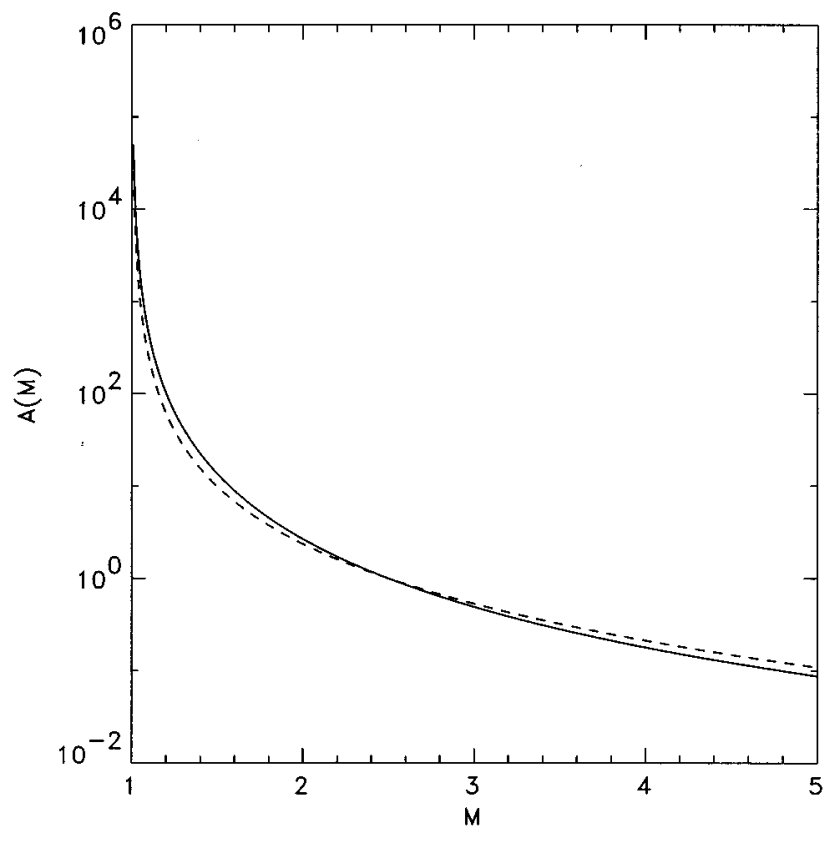

FIG. 2. Comparison of the area-Mach number relation $A(M)$ for water using the modified Tait equation $\Gamma=7.15$ and perfect gas relation, $\gamma=1.4$.

$$
\begin{aligned}
A(M)= & \exp \left[\int_{M_{0}}^{M} \frac{1}{M}\left(\frac{R^{(\Gamma+1) / 2}+M}{R^{(\Gamma+1) / 2}-M}\right)\right. \\
& \left.\times\left(1+\frac{M(R-1)}{R^{(\Gamma+1) / 2}}\right) d M\right] .
\end{aligned}
$$

A comparison of the $A(M)$ relation for water using the modified Tait equation with $\Gamma=7.15$ and the perfect gas relation with $\gamma=1.4$ is shown in Fig. 2. The reference Mach number $M_{0}$ for the $A(M)$ relation is taken to be the midpoint of the Mach number range, i.e., the areas at the midpoint are set to be equal. It is seen that $A(M)$ for a perfect gas with $\gamma=1.4$ and water with $\Gamma=7.15$ are nearly the same. Thus in this paper we report only results obtained with the perfectgas equation of state.

The equations of geometrical shock dynamics form a hyperbolic, second-order partial differential equation that describes the wave motion of disturbances propagating along the shock front. Discontinuities, called shock-shocks, can form along the shock carrying a change in shock angle and an increase in Mach number. Shock-expansions can also form, for example in shock diffraction around a corner.

In this paper an improved finite-difference scheme is developed based on the methods developed for the supersonic potential equation, ${ }^{17}$ which is suitable for weak-shock problems with strong discontinuities. Introducing an arbitrary coordinate system (not necessarily orthogonal) defined by $\xi=\xi(x, y), \eta=\eta(x, y)$, Eq. (2.1) can be written in strongconservation form:

$$
\frac{\partial}{\partial \xi}\left(\frac{\sigma U}{J}\right)+\frac{\partial}{\partial \eta}\left(\frac{\sigma V}{J}\right)=0,
$$

where $U, V$ are the contra-variant velocity components given by 


$$
\begin{aligned}
& U=a_{11} \alpha_{\xi}+a_{12} \alpha_{\eta}, \quad V=a_{12} \alpha_{\xi}+a_{22} \alpha_{\eta}, \\
& \sigma=\frac{M}{A(M)},
\end{aligned}
$$

and the grid parameters are

$$
\begin{aligned}
& a_{11}=\xi_{x}^{2}+\xi_{y}^{2}, \quad a_{12}=\xi_{x} \eta_{x}+\xi_{y} \eta_{y}, \quad a_{22}=\eta_{x}^{2}+\eta_{y}^{2} \\
& J=\xi_{x} \eta_{y}-\xi_{y} \eta_{x} .
\end{aligned}
$$

The Mach number is now given by

$$
M=\left[U \alpha_{\xi}+V \alpha_{\eta}\right]^{-1 / 2} \text {. }
$$

In the solution procedure, we consider $\xi$ to be the marching, time-like direction and assume that all information is known at the $i$ th and all previous levels. The problem is to advance the solution to the level $i+1$ and obtain new $\alpha$ values.

At all levels, the quantities $\alpha_{\xi}, \alpha_{\eta}$ are computed from

$$
\left(\alpha_{\xi}\right)_{i, j}=\frac{\alpha_{i, j}-\alpha_{i-1, j}}{\Delta \xi} ; \quad\left(\alpha_{\eta}\right)_{i, j}=\frac{\alpha_{i, j+1}-\alpha_{i, j-1}}{2 \Delta \eta},
$$

where a backward difference is used for the marching direction $\xi$ and a central difference is used for the cross-flow direction $\eta$. For half-points, the velocities are averaged between neighboring points which gives

$$
\begin{aligned}
\left(\alpha_{\xi}\right)_{i, j+1 / 2} & =\frac{\left(\alpha_{i, j+1}-\alpha_{i-1, j+1}\right)+\left(\alpha_{i, j}-\alpha_{i-1, j}\right)}{2 \Delta \xi}, \\
\left(\alpha_{\eta}\right)_{i, j+1 / 2} & =\frac{\alpha_{i, j+1}-\alpha_{i, j}}{\Delta \eta} .
\end{aligned}
$$

Once these quantities are known, $U$ and $V$ follow directly from Eq. (2.11) and $M$ from Eq. (2.15).

Since the $\xi$ direction is the marching direction, the $\xi$ derivative in Eq. (2.10) is backward differenced as

$$
\frac{\partial}{\partial \dot{\xi}}\left(\frac{\sigma U}{J}\right)_{i+1, j} \doteq \frac{\left(a_{1}-\theta b_{1}\right)\left\{(\sigma U / J)_{i+1, j}-(\sigma U / J)_{i, j}\right\}-\theta b_{1}\left\{(\sigma U / J)_{i, j}-(\sigma U / J)_{i-1, j}\right\}}{a_{1} \Delta \xi_{1}-\theta b_{1}\left(\Delta \xi_{1}+\Delta \xi_{2}\right)}
$$

where

$$
a_{1}=\left(\Delta \xi_{1}+\Delta \xi_{2}\right)^{2}, \quad b_{1}=\left(\Delta \xi_{1}\right)^{2},
$$

and

$$
\Delta \xi_{1}=\xi_{i+1}-\xi_{i}, \quad \Delta \xi_{2}=\xi_{i}-\xi_{i-1} .
$$

The parameter $\theta$ controls the order: $\theta=0$ gives first-order accuracy and $\theta=1$ gives second-order accuracy. ${ }^{17}$

The upwind differencing of the $\xi$ derivative term produces a truncation error whose leading term is

$$
\frac{\sigma}{J a^{2}}\left(1-\frac{a^{2} a_{11}}{U^{2}}\right) U^{2} \alpha_{\xi \xi \xi} \Delta \xi .
$$

This term represents a positive artificial viscosity if

$$
\frac{U^{2}}{a_{11}}>a^{2}
$$

In general the stability requirement, Eq. (2.23), is satisfied if the information propagates along characteristics only from behind the current point. To march in the $\xi$ direction, the domain of dependence for point $i, j$ must not include information from forward of the current $\xi$ row. For problems where the shock is strongly curved, the grid must be chosen to maintain $\xi$ as the propagation direction. For weak shocks, the characteristic angle approaches zero and stability requires only that the component of the shock velocity along the marching direction be positive. Stability is more restrictive for large Mach number, as the characteristic angle increases, and the $\xi$ direction must be more closely aligned with the shock normal. Ideally, the grid should be aligned such that locally at each point the $\xi$ direction is normal to the shock. The stability requirement Eq. (2.23), was checked at each point in the calculations.

The $\eta$ derivative term in Eq. (2.10) is centraldifferenced and written at level $i+1$ to make the resulting scheme fully implicit,

$$
\frac{\partial}{\partial \eta}\left(\frac{\sigma V}{J}\right)_{i+1, j} \doteq \frac{1}{\Delta \eta}\left\{\left(\frac{\sigma V}{J}\right)_{i+1, j+1 / 2}-\left(\frac{\sigma V}{J}\right)_{i+1, j-1 / 2}\right\} .
$$

The suitability of the above difference depends on the nature of the flow in the cross-flow plane. When $1-a_{22} a^{2} / V^{2}<0$, the cross flow is elliptic, as shown in Fig. 3(a). The characteristics propagate information from both the positive and negative $\eta$ directions, and central differencing is appropriate. For hyperbolic cross flow as shown in Fig. 3(b), $1-a_{22} a^{2} / V^{2}<0$. In this case, the characteristics propagate

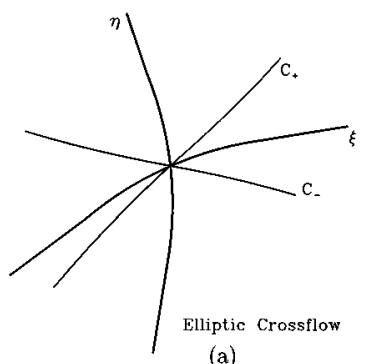

(a)

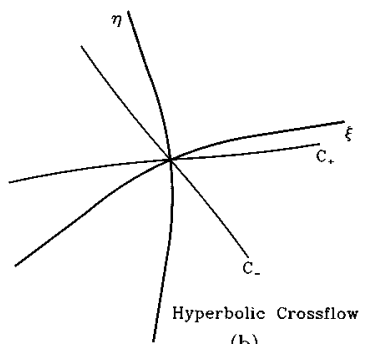

(b)
FIG. 3. Cross-flow type according to characteristic angles. 
information in a single $\eta$ direction, and the central differencing of Eq. (2.24) is unstable. In this case, artificial viscosity is necessary to preserve stability.

To implement the required artificial viscosity, the value of $\sigma$ is biased in the direction of the cross flow $V$. This corresponds to density biasing, often referred to as artificial compressibility in the case of the supersonic potential equation. The $\sigma$ in Eq. (2.24) is replaced by $\widetilde{\sigma}$,

$$
\begin{aligned}
\widetilde{\sigma}_{i+1, j+1 / 2}= & (1-\nu) \sigma_{i+1, j+1 / 2}+\frac{1}{2} \nu\left(\sigma_{i+1, j+1 / 2+2 k}\right. \\
& \left.+\sigma_{i+1, j+1 / 2-1}\right),
\end{aligned}
$$

where $k=0$ when $V_{i+1, j+1 / 2}>0$ and $k=1$ when $V_{i+1, j+1 / 2}<0$. The artificial viscosity $\nu$ utilized in this implementation employs upwind differencing based on the direction of the characteristic signal propagation; when the cross flow is elliptic the upwind differencing is turned off, ${ }^{17}$

$$
\nu_{i+1, j+1 / 2}=\zeta\left[1-\frac{a_{22} a^{2}}{V^{2}}\right]_{i+1, j+1 / 2},
$$

with

$$
\begin{aligned}
& \zeta=0, \text { for }\left(a_{22}-\frac{V^{2}}{a^{2}}\right)_{i+1, j+1 / 2}>0 \\
&(\text { elliptic cross flow), } \\
&=1 \quad \text { for }\left(a_{22}-\frac{V^{2}}{a^{2}}\right)_{i+1, j+1 / 2}<0
\end{aligned}
$$

(hyperbolic cross flow).

Given the $\alpha$ values at all previous levels, the differencing results in a set of nonlinear algebraic equations for $\alpha_{i+1}$ which are solved using Newton's method. In general, the artificial viscosity leads to a pentadiagonal system. For large Mach numbers, when the artificial viscosity terms are small, and for flows without strong shock-shock discontinuities the derivative terms due to the artificial viscosity can often be neglected, yielding a tridiagonal system. In this case, more iterations may be required for convergence of each $\xi$ step, but each iteration is significantly faster.

The boundary condition of shock dynamics requires that the shock front be normal to solid boundaries or axes of symmetry. If $\mathbf{n}$ is the unit vector normal to the boundary, the condition requires $\partial \alpha / \partial n=0$ at the boundary. For the grid systems used in this work, the boundary is given by $\eta=$ const; therefore, the boundary condition reduces to $V=0$. The initial conditions $\left(\alpha_{0}, M_{0}\right)$ are specified along an initial row, $\xi=$ const. Since the Mach number specifies the gradient of $\alpha$, the value of $\alpha$ at the previous $\xi$ can be estimated from the Mach number. Then, with $\alpha$ values specified for two initial $\xi$ rows, the solution may be advanced.

\section{FOCUSING RESULTS}

The convergence of a segment of a cylindrical shock front to a perfect line focus is considered. This problem is characterized by two parameters shown in Fig. 4, the initial

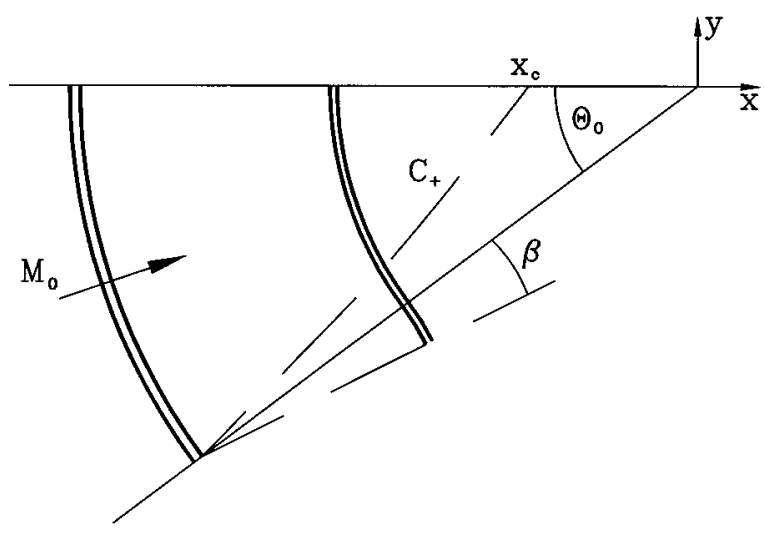

FIG. 4. Focusing problem parameters: initial shock Mach number $M_{0}$ and the aperture angle $\theta_{0}$.

Mach number $M_{0}$ and the aperture angle $\theta_{0}$. At the very least, to be judged successful a numerical model should duplicate the strong-, moderate- and weak-focusing behavior observed in experiments. ${ }^{1}$ This requires the prediction of the path of the experimentally observed triple point, represented by a shock-shock in the present analysis. The path of the triple point is the most demanding element, since this defines the geometry of the flow near the focus. Other important parameters for judging the validity of a simulation include the shock front positions downstream of the focus the shock pressure jump at focus and the location of the maximum pressure jump. Conditions behind the leading shock cannot be determined by the theory of shock dynamics.

In shock dynamics the angle $\beta$ through which a shock of initial strength $M_{0}$ can be turned in an expansion before its strength vanishes is finite. This means that for weak shocks the bottom of our computational domain can not be horizontal. In such cases the lower boundary was placed at an angle slightly larger than $\theta_{0}-\beta$ to the horizontal (Fig. 4) to arrange for the shock front at the corner to be turned to a Mach number approaching unity.

For all cases, typical runs used several hundred grid points in the $\eta$-direction. For better resolution of the focal region, grid points were packed near the upper boundary. The solution was marched at variable $\xi$ steps chosen to maintain an average CFL number of unity. Runs of varying mesh density were made to verify that the results were independent of grid resolution, except locally in some cases mentioned below. Computations of the focusing of moderate-tostrong shock waves $\left(M_{0}=1.5-2.0\right)$ typically take several minutes on an $85 \mathrm{MHz}$ workstation, while weak shocks, with increased grid density required near the axis, slower convergence for very strong shock-shocks, and small time steps to insure stability, can take as long as 10-20 min. In this paper we present detailed results for strong and moderately strong shock waves $\left(M_{0}=1.3\right.$ and 1.1). Calculations for weaker waves show that the behavior is the same as for the $M_{0}=1.1$ case (a shortcoming of shock dynamics theory discussed below), except that the scale of the focal zone decreases rapidly with decreasing shock strength. Therefore, we do not present the complete results for weak-shock cases, 


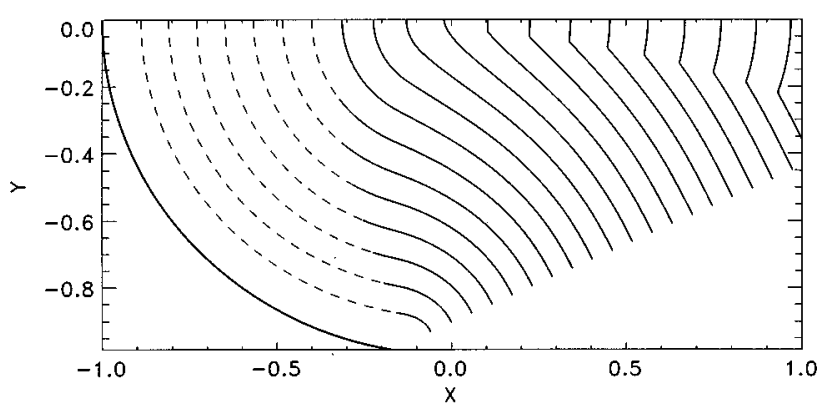

(a)

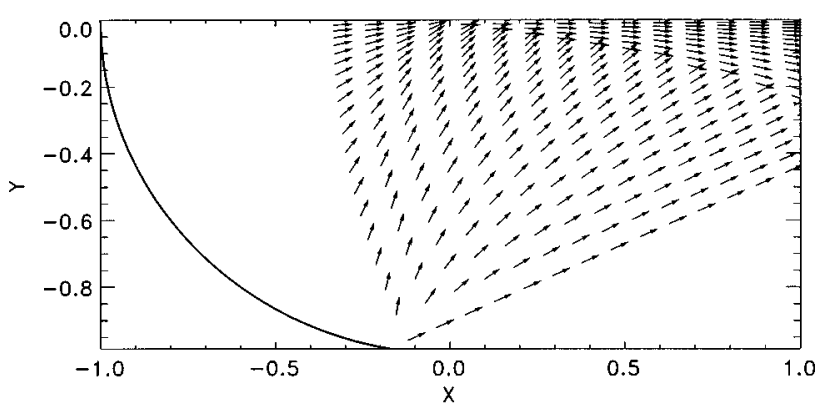

(b)

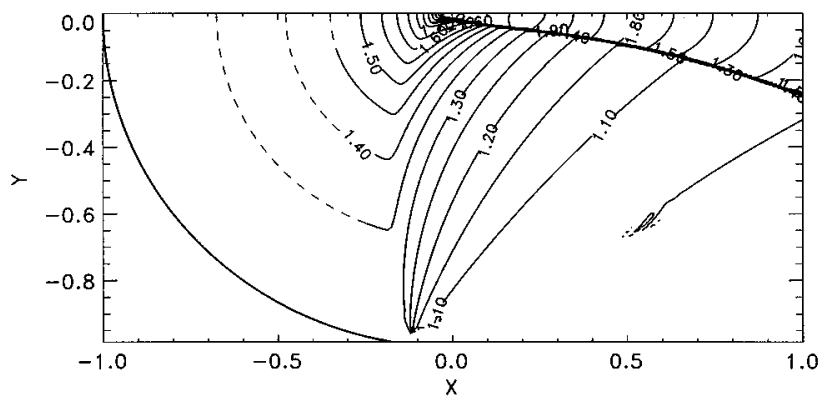

(c)

FIG. 5. (a) Shock fronts, (b) shock front normals and (c) Mach number contours for converging shock, $M_{0}=1.3, \theta_{0}=80^{\circ}, \gamma=1.4$. The center of the initial front lies at $(x, y)=(0,0)$.

but we plot centerline pressures and maximum wave strengths, together with the location at which they occur, for all cases computed, $1.01<M_{0}<1.3$.

\section{A. Results for $80^{\circ}$ aperture angle}

Results for aperture angle $\theta_{0}=80^{\circ}$ and initial Mach number $M_{0}=1.3$ are shown in Figs. 5 and 6. Figure 5(a) shows contours of constant phase $\alpha$. The normals to the fronts are shown in the ray diagram, Fig. 5(b). Figure 5(c) displays Mach number contours. A surface plot of the shock Mach number is shown in Fig. 6. For clarity, the solution at every grid point is not plotted on the surface plot. This has the effect that the discontinuity appears sharper than actually calculated.

This Mach number-aperture combination is an example of strong-shock behavior. The leading characteristic from the expansion corner at the lower end of the initial shock, and the shock-expansion behind it turn the shock front away from the centerline, thereby slowing the increase of the Mach

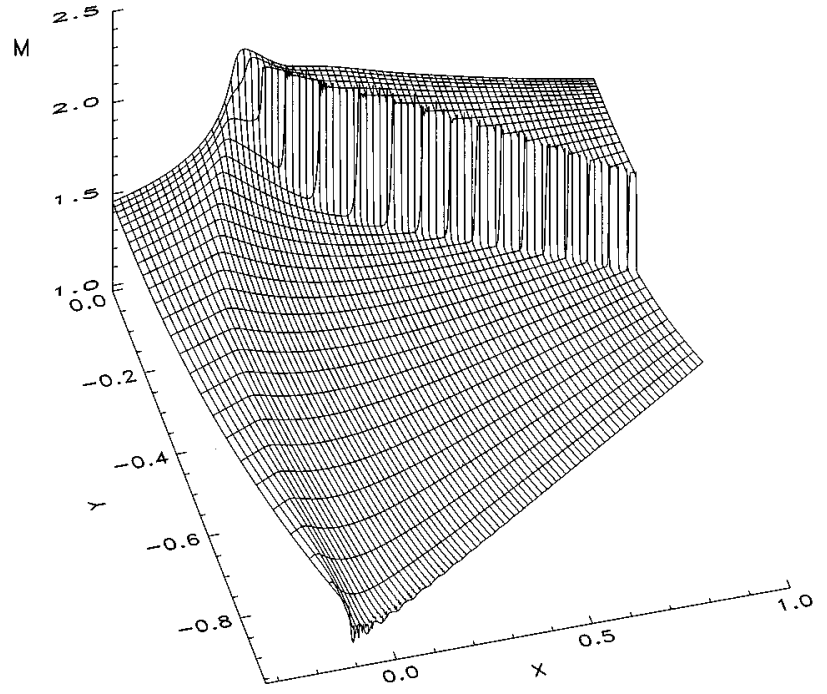

FIG. 6. Mach number surface for converging shock, $M_{0}=1.3, \theta_{0}=80^{\circ}$, $\gamma=1.4$. The center of the initial front lies at $(x, y)=(0,0)$. A slight overshoot is visible at the upper-edge of the shock-shock discontinuity.

number. To maintain the boundary condition of shockdynamics theory that the shock front be normal to the centerline, the reflection of the shock-expansion from the centerline turns the front back toward the centerline. These reflected disturbances are compressive, and eventually coalesce into a shock-shock discontinuity. The shock-shock is clearly shown in the figures by a change of shock front angle [Figs. 5(a) and (b)] and an increase of Mach number [Figs. 5(a) and 6]. It moves continuously outward from the centerline. As the Mach stem extends it becomes slightly convex forward and it weakens.

A quantitative measure of the shock-shock location is derived by plotting the trajectory of the maximum gradient of Mach number on each solution row (Fig. 7). From the left, the maximum gradient shows the path of the expansion from the lower corner, and after reflection it shows the path of the shock-shock. For strong shock waves [Fig. 7(a)] the shockshock moves continuously outward after focus, consistent with the description of Sturtevant and Kulkarny ${ }^{1}$ [Fig. 1(d)].

An example of moderate shock behavior $\left(M_{0}=1.1\right.$, $\theta_{0}=80^{\circ}$ ) is shown in Figs. 8 and 9. The maximum turning angle for $M_{0}=1.1$ is $51^{\circ}$, so the lower boundary must again be inclined to the centerline. In this case the shockexpansion reflected from the axis of symmetry almost immediately coalesces into a shock-shock discontinuity. Although the triple point propagates outward along the shock front, the shock front itself is still directed strongly inward. The net result is that the shock-shock discontinuity is swept back toward the centerline [Fig. 7(b)]. The strength of the shockshock increases as it is pushed inward, producing a further increase in the centerline Mach number. The inward motion of the shock-shock forms a shoulder in the Mach number profile, shown in the Mach number surface plot, Fig. 9. The strength of the shock-shock continues to increase until its velocity along the shock front is sufficient for it to move outward. Downstream of the focus, a narrow Mach-stem is visible. 


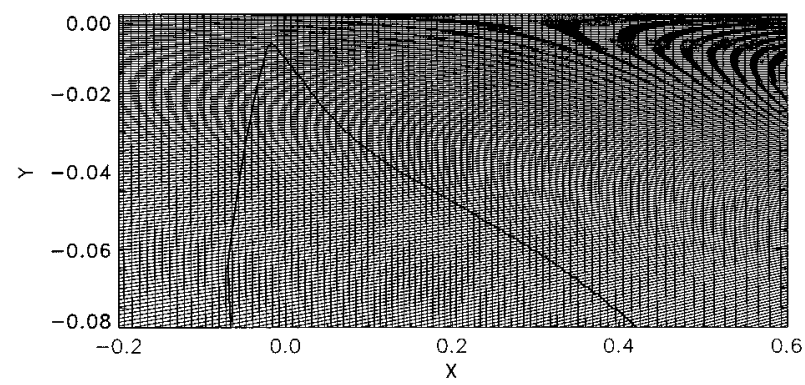

(a)

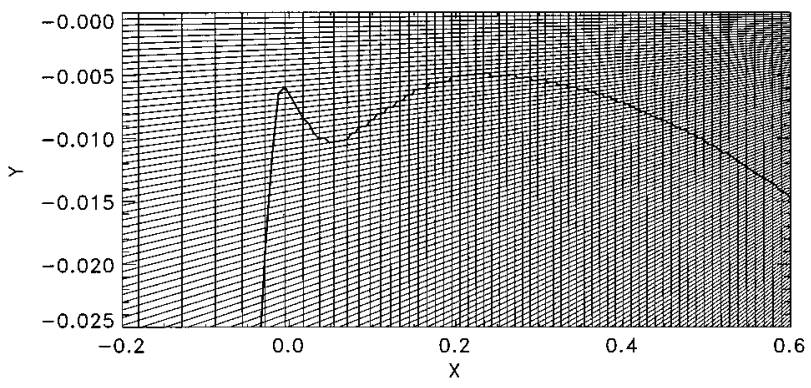

(b)

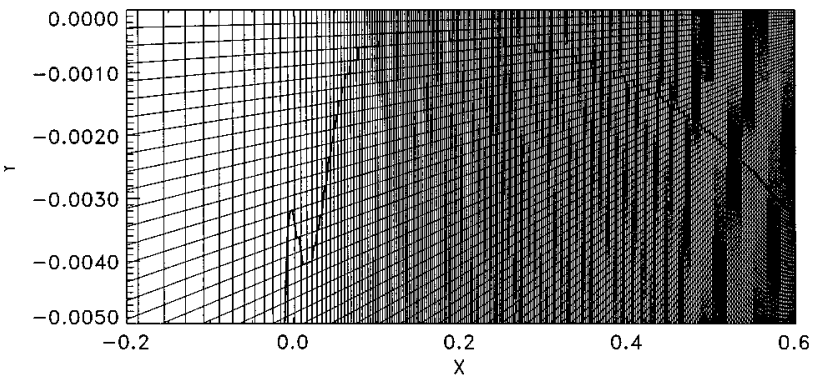

(c)

FIG. 7. Location of the maximum Mach number gradient near the focus for strong, $M_{0}=1.3$, moderate, $M_{0}=1.1$, and weak, $M_{0}=1.05$, shock behavior for $\theta_{0}=80^{\circ}, \gamma=1.4$ superimposed over the solution grid. For weak shock behavior, the discontinuity is immediately pushed to within several grid points of the centerline.

Figure 7(b) shows that the behavior of moderate strength focusing shocks is in qualitative agreement with the findings of Sturtevant and Kulkarny ${ }^{1}$ [Fig. 1(c)]. Adequate grid points exist to correctly capture the distance of the discontinuity from the wall at the closest approach. The distance between the arrival of the expansion disturbance from the lower corner and the point of closest approach of the shock-shock defines the size of the "focal region.",

Calculations show that weaker shocks behave in the same way. The disturbance from the lower corner arrives at the axis closer to the acoustic focus than for stronger shocks and a shock-shock forms at the focus. The weaker the shock wave the stronger is the shock-shock that forms. The discontinuity is pushed back toward the centerline almost immediately and the focal region gets continually smaller as the Mach number decreases. For weak shocks, the shock-shock is actually pushed to within several grid points of the centerline [Figure 7(c)], and the path of the shock-shock in the region near the closest approach becomes dependent on the

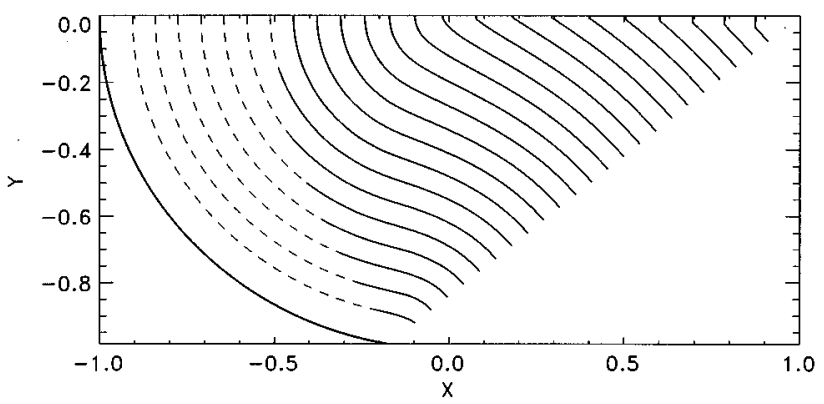

(a)

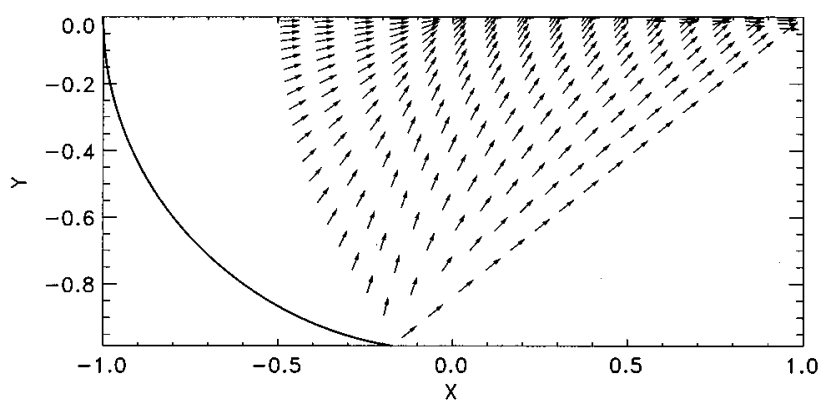

(b)

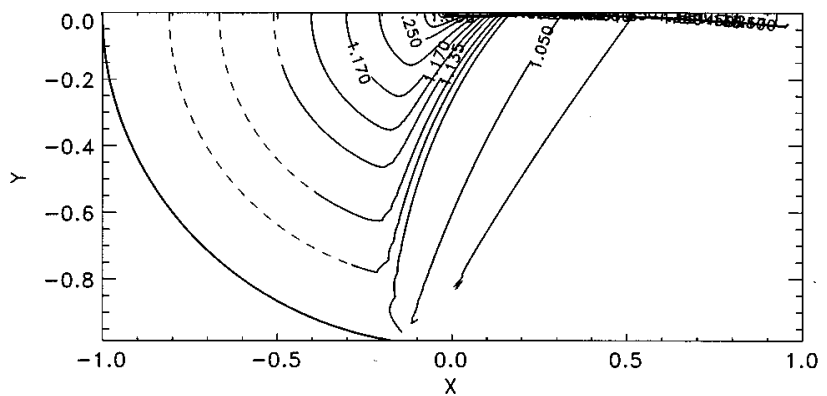

(c)

FIG. 8. (a) Shock fronts, (b) shock front normals and (c) Mach number contours for converging shock, $M_{0}=1.1, \theta_{0}=80^{\circ}, \gamma=1.4$. The center of the initial front lies at $(x, y)=(0,0)$.

grid spacing. In this case the Mach stem is not resolved, and for all practical purposes the reflection at the axis of symmetry is regular there. This is consistent with experimental findings that the post-focus configuration for weak shocks is regular reflection, as predicted by geometrical acoustics. ${ }^{1}$ However, because of the normal-shock boundary condition of shock dynamics theory, the shock-shock inevitably strengthens and the Mach stem lengthens as the shock propagates downstream, and for all shock strengths calculated in this work the shock-shock eventually departs from the neighborhood of the axis, in disagreement with the findings of experiments.

Figure 10 plots the shock pressure on the axis of symmetry for strong $\left(M_{0}=1.3\right)$, moderate $\left(M_{0}=1.1\right)$ and weak shocks $\left(M_{0}=1.05\right)$ for aperture angle $\theta_{0}=80^{\circ}$. The $x$-coordinate is nondimensionalized with the initial radius, and the geometrical focus lies at $x=0$. The pressure is normalized with the initial strength of the shock wave $p_{0}\left(M_{0}\right)$ obtained from the Rankine-Hugoniot relations for a perfect 


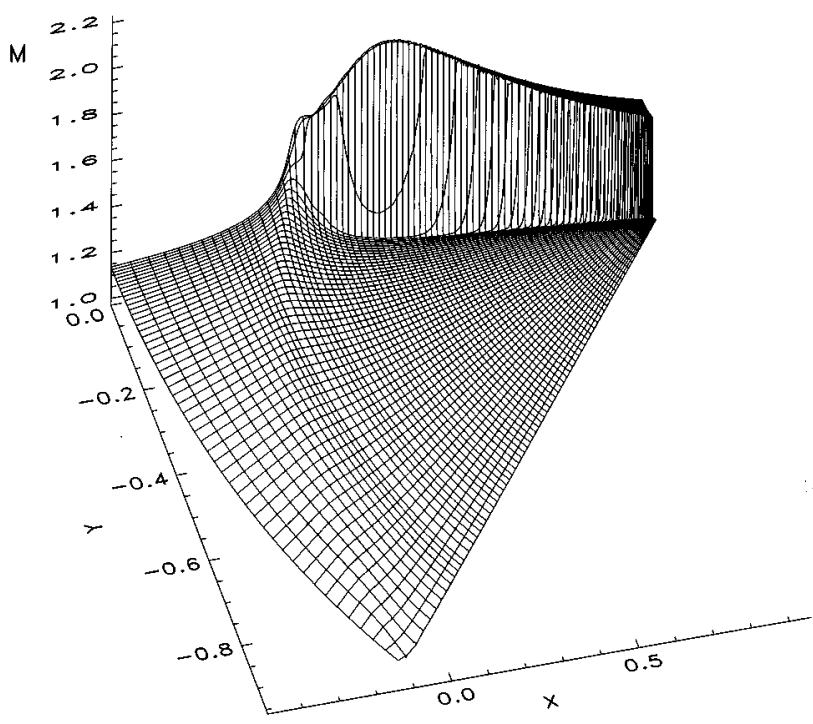

FIG. 9. Mach number surface for converging shock, $M_{0}=1.1, \theta_{0}=80^{\circ}$, $\gamma=1.4$. The center of the initial front lies at $(x, y)=(0,0)$.

gas. For strong shocks a single pressure peak is formed ahead of the acoustic focus. For moderate-strength shocks a local maximum occurs before the focus when the lead expansive disturbance from the lower end of the initial shock front reaches the centerline. An absolute maximum occurs farther downstream when the shock-shock becomes strong enough to again move outward. For the weak case $\left(M_{0}=1.05\right)$ the upstream shoulder is visible only as a change of slope.

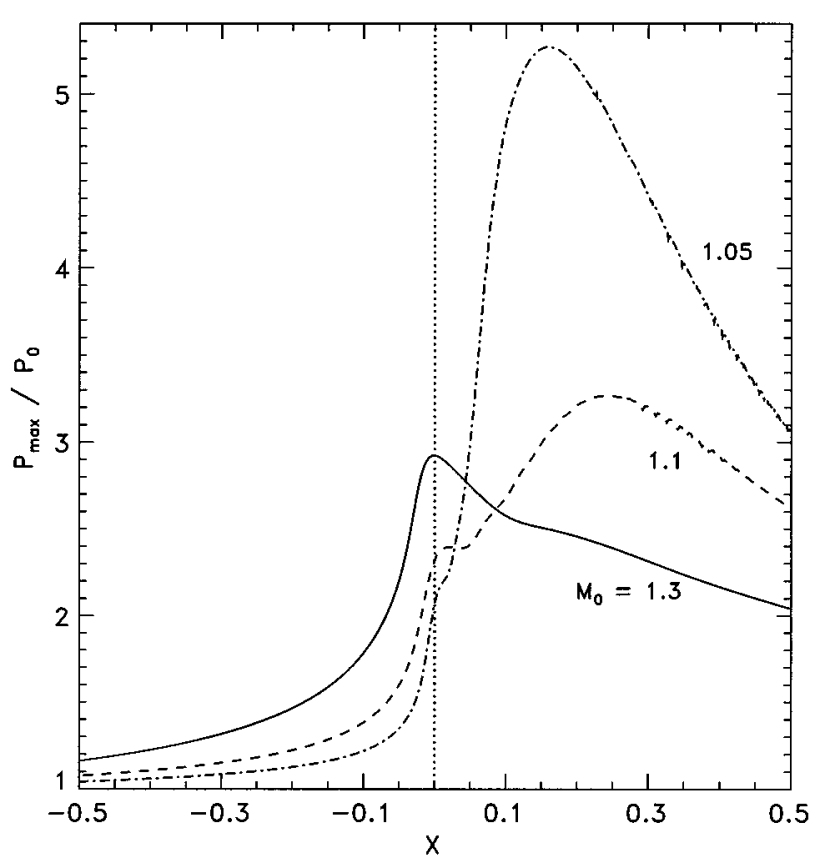

FIG. 10. The ratio of the calculated shock pressure along the axis of symmetry to the initial shock pressure versus the distance from the geometrical focus for three different initial Mach numbers, $M_{0}$. The shock propagates from left to right and the pressures are measured at $y=0$ in Fig. 4. Aperture angle $\theta_{0}=80^{\circ}, \gamma=1.4$.

\section{B. Effect of aperture angle}

A similar set of cases was computed for aperture angle $\theta_{0}=36^{\circ}$, corresponding to the reflector ellipse used in the modified Dornier HM3 lithotriptor which has an eccentricity $e=0.8$. Two major effects of the smaller aperture angle are seen in the results. The expansion disturbance from the lower corner has less transverse distance to travel and reaches the centerline to start the focusing process farther upstream. This also means that the expansion reaches the centerline when the shock strength is smaller, producing lower peak pressure at the focus. Due to the smaller Mach number, the onset of weak shock behavior occurs at a smaller Mach number. For $\theta_{0}=36^{\circ}$, moderate-strength behavior is still seen at $M_{0}=1.1$, while weak-shock behavior is seen at $M_{0}=1.01$.

A summary of the maximum Mach number and its location for the two aperture angles $\left(\theta_{0}=36^{\circ}\right.$ and $\left.80^{\circ}\right)$ is shown in Fig. 11. The open points indicate the maximum Mach number computed on the axis of symmetry for each case. The pluses indicate the lower Mach number (and the corresponding location) at the shoulder described above (Figs. 9 and 10) for moderate-strength and weak shock waves. As described below, the pressure at the shoulder agrees better with the amplitude and location of experimental maximum pressures than does the absolute maximum computed pressure. The dashed lines in Fig. 11 show the Mach number and position of the shock when the leading disturbance from the lower end of the initial front reaches the axis of symmetry. The results show that the arrival of the lead disturbance from the lower corner provides an accurate estimate of the beginning of the focal region. The absolute maximum of Mach number occurs farther downstream as the bulk of the expansive disturbance from the lower corner of the initial front arrives.

For aperture angle $\theta_{0}=36^{\circ}$, a relatively shallow reflector with relatively larger effects of nonlinearity, the focus occurs ahead of the acoustic focus, except for the very weak case, $M_{0}=1.01$. For this case, even the initial shoulder forms slightly behind the acoustic focus. For $\theta_{0}=80^{\circ}$, a deep reflector with smaller nonlinearity, the location of the focus is behind the acoustic focus for weaker shocks. The focus Mach number is larger with the large aperture angle, for all initial Mach numbers.

\section{COMPARISON WITH EXPERIMENTAL RESULTS}

Numerical shock dynamics was used to simulate the focusing experiments of Sturtevant and Kulkarny. ${ }^{1}$ In this series of experiments, plane shock waves were reflected from a parabola to a line focus. After reflection, the shock propagated back into the uniform flow behind the incident shock. This problem can be treated with a generalization of the method presented here. ${ }^{18}$ However, since the velocity is uniform, a simple coordinate transformation is sufficient. If the uniform velocity is given by $u_{0}$, the free stream is at rest in the coordinate frame given by $x^{\prime}=x-u_{0} t$. Therefore, at any point on the shock front $\alpha\left(\mathbf{x}^{\prime}\right)=a_{0} t$, the physical $x$-coordinate value must be computed as 

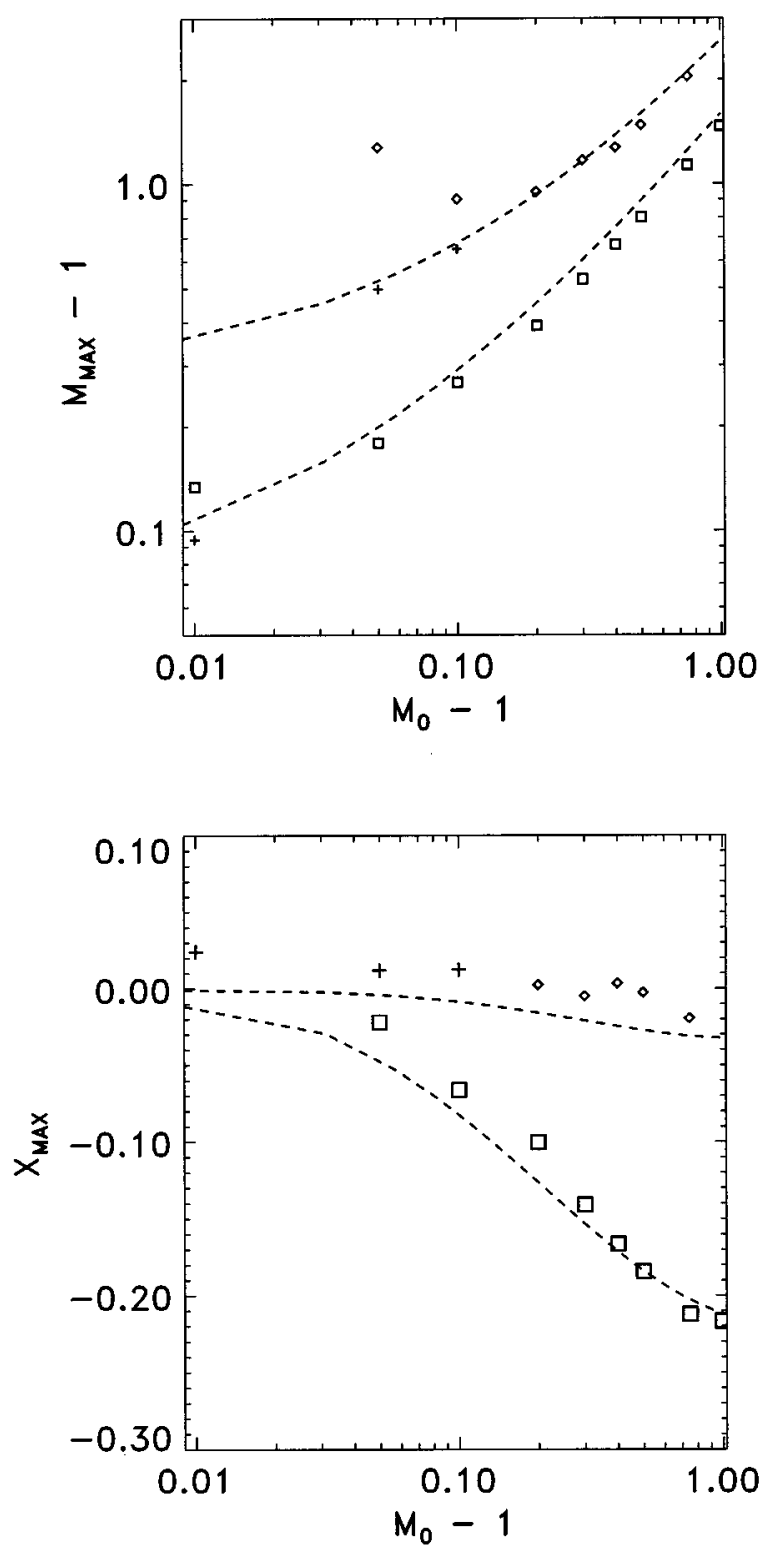

FIG. 11. The maximum Mach number on the axis of symmetry and the $x$-location of the maximum for all shock strengths computed. Aperture angles $\theta_{0}=80^{\circ}(\diamond)$ and $\theta_{0}=36^{\circ}(\square)$. ( + ), shock Mach number and location of the shoulder on the centerline pressure history (Fig. 10) which appears for moderate and weak shock strengths. Dashed lines represent the shock Mach number and location at the point when the lead disturbance from the end of the initial front arrives at the axis of symmetry. Perfect gas, $\gamma=1.4$.

$$
x=x^{\prime}+\left(\frac{u_{0}}{a_{0}}\right) \alpha .
$$

Provided that the initial conditions are specified properly in the $\left(x^{\prime}, y\right)$ coordinate system, the problem can be solved with the previous numerical scheme.

A parabolic grid was used for the calculation so the parabolic reflector is a line of constant $\xi$, and the initial conditions are specified along the reflector. The initial $\alpha$ values were calculated from the arrival time of the incoming shock at each point on the reflector. The Mach number was taken to be the Mach number of the initial planar shock. Computations using exact values of the reflected-shock Mach number showed that the slight variation in initial Mach number along the reflector had little effect on the focal region.

The computed pressure amplification and shock-shock path are compared with the experimental results in Fig. 12. The pressure amplification is defined as the pressure jump across the shock normalized by the pressure jump of the reflected shock as it leaves the reflector surface. In the experimental results, the maximum pressure outside the focal region occurred downstream of the shock, and is not the pressure computed by shock dynamics theory. However, within the focal region, the maximum experimental pressure occurred at the shock front and direct comparison can be made with the results of shock dynamics. For the three cases shown in Fig. 12(a) shock dynamics theory overestimates the maximum pressure by $60 \%-90 \%$. The over-prediction is not significantly affected by accounting for the finite size of the pressure transducer used in the experiments. Since the comparison is to ratios of measured pressures, the errors in the experimental results are probably an order of magnitude smaller than this discrepancy. For strong shocks the location of the computed maximum pressure agrees with experiment, but when a shoulder occurs in the computed variation of pressure with distance in the intermediate and weak-shock cases, the location of the shoulder accurately records the experimentally observed location of maximum pressure. If the pressure amplitude computed at the shoulder is taken to be the focal pressure, then the overestimate reduces from $90 \%$ to $55 \%$ for the $M_{0}=1.1$ case. The additional rise of Mach number in these cases, due to the requirement for Mach reflection at the centerline, is not seen experimentally.

As discussed above, the path of the shock-shock depends sensitively on the interplay between several nonlinear effects, so it is one of the most exacting tests that can be made of computational results. In Fig. 12(b) the path is reasonably well predicted for $M_{0}=1.3$, but for weaker shocks the agreement deteriorates. Although moderate-strength behavior (focal region of finite length) was experimentally observed for $M_{0}=1.2$, shock dynamics does not show this behavior until the Mach number reduces to $M_{0}=1.1$. For all cases, the origin of the shock-shock agrees closely with the origin of the triple point observed in the experiments.

\section{DISCUSSION}

In this work numerical solutions of shock wave focusing by geometrical shock dynamics theory have been used to examine nonlinear gasdynamic behavior near foci and to test the shock dynamics approximation in a difficult problem. The fact that shock dynamics theory yields hyperbolic equations for the behavior of disturbances on the shock front permits a physical interpretation of the results in terms of the characteristic curves of the equations. In shock focusing, diffracted waves from the edges of the aperture limit the amplitude at focus and determine the geometry of the focal field. The arrival at the centerline of the leading expansive disturbance from the lower corner of the initial front signals the beginning of the process which limits infinite convergence of the shock discontinuity. For weak shocks the speed of the diffracted disturbance along the shock front is small, so it does not reach the centerline until near the acoustic focus and 


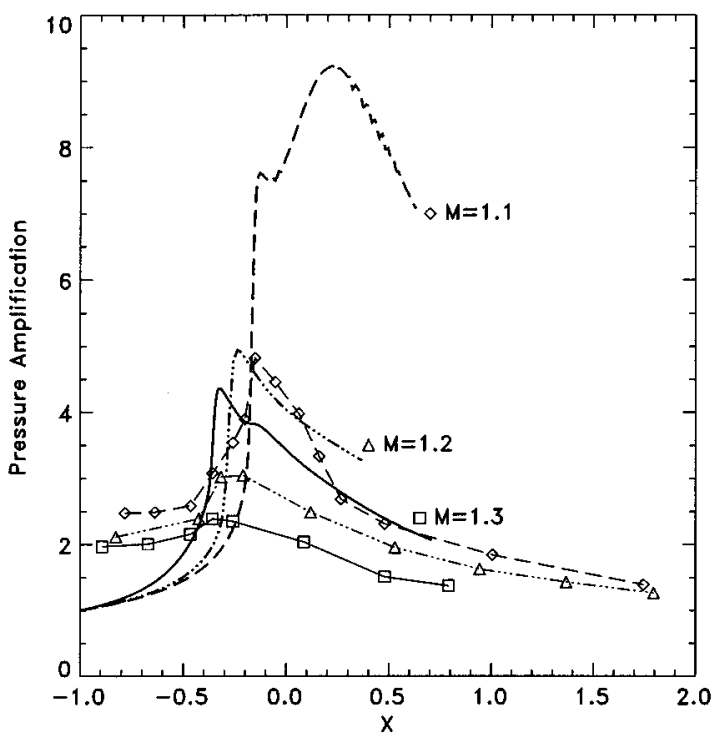

(a)

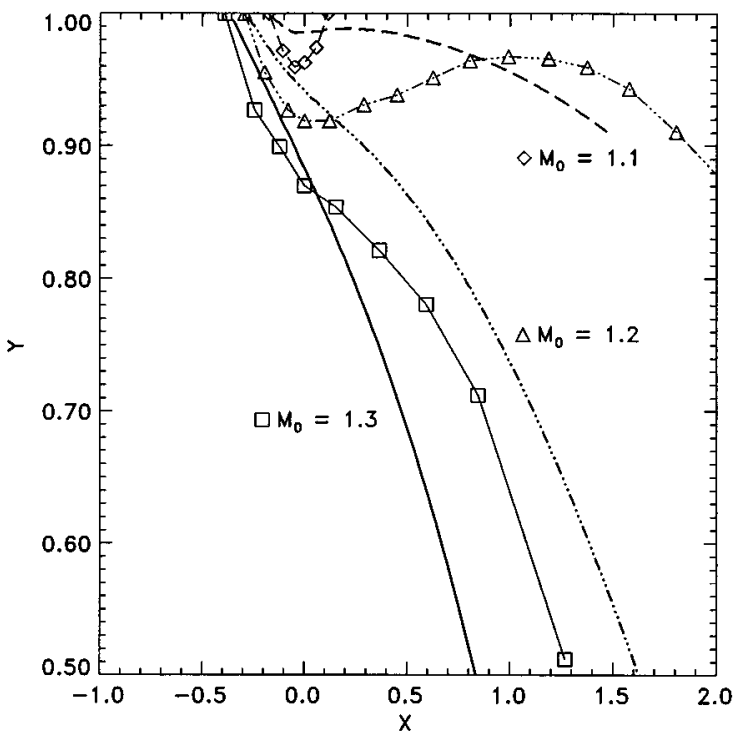

(b)

FIG. 12. (a) Pressure amplification and (b) shock-shock path computed for the reflection of planar shock waves from a parabola compared with experimental results of Sturtevant and Kulkarny (1976). The numerical results are shown as lines without symbols, experimental results are corresponding line types with symbols. The experimental pressure amplification is based on the maximum pressure which outside the focal region is not the pressure directly at the shock.

the amplification is large. In the limit $M_{0}=1$ the disturbance reaches the centerline precisely at the geometric focus, the extent of the focal region shrinks to zero and the singularity of acoustic theory is recovered. For finite-strength shocks the nonlinear propagation velocity of disturbances along the shock is larger, so the first disturbance always arrives at the centerline before the acoustic focus. Likewise, for smaller apertures the disturbance has a shorter transverse distance to travel to reach the centerline so diffraction effects are felt further upstream of the acoustic focus and at a lower Mach number, i.e., nonlinear effects are larger.

In an analysis of weak shocks diffracting around a corner with a small turning angle Whitham ${ }^{6}$ pointed out that the speed of the initial signal predicted by shock dynamics is half the value given by first-order gasdynamics theory. In the current problem this shortcoming results in the leading disturbance from the lower corner arriving at the centerline too late, at which time the focusing has developed too far, and the resulting shock-shock is too strong and has too large a speed outward from the centerline. This explains both the over-prediction of the pressure at focus and the excessively wide focal region compared to experimental results.

For very weak shocks, the computations show that the focal region closes downstream, in agreement with experiments, in the sense that the shock-shock returns to within a few mesh points of the axis of symmetry. We emphasize that a method without adequate resolution would not detect this effect; all shocks would appear to be "strong." However, even with adequate resolution, no matter how small the Mach number (for $M_{0}>1.01$ ), if the computation is run long enough the shock-shock eventually departs from the axis and becomes resolved. This occurs because of the parallelflow boundary condition of shock dynamics. A consequence is that the strength of the shock-shock increases as it is pushed back toward the axis of symmetry in weak-shock focusing, until its velocity along the shock front inevitably becomes so large that it again moves outward. Focusing experiments ${ }^{1}$ do not indicate that, once the wavefronts cross and fold in regular reflection about the axis of symmetry, a Mach reflection configuration ever reappears. However, in other diffraction problems transition from regular to Mach reflection about an axis of symmetry is common (see Haas and Sturtevant, ${ }^{19}$ Fig. 11(f), and Bryson and Gross ${ }^{12}$ ), so it can not be ruled out that at least for some focusing configurations this could occur. Thus, it is not certain whether the computed departure of the shock-shock from the axis is simply a premature manifestation of an effect which might eventually occur in the experiments further downstream, or whether it is a fundamental failure of shock dynamics theory in treating the focusing of very weak shock waves.

For a fair comparison with experimental results, it is important that the focal region be adequately resolved numerically. Too coarse a grid or too high an artificial viscosity reduces the maximum Mach number at the focus, leading to an over-optimistic assessment of the validity of shock dynamics for shock-focusing problems. This is especially true for weak shocks, where the focal region becomes very small and extremely sensitive to grid resolution.

In this paper examples from computations for a perfect gas with $\gamma=1.4$ have been presented. In the shock wave lithotripsy shock waves are generated in water and transmit into the patient's body, also a water-like substance. We have shown (Fig. 2) that the behavior in water modeled by the modified Tait equation [Eq. (2.5)] is similar to that in a perfect gas, as might be expected because $\Gamma$ in the Tait equation plays the same role as $\gamma$ in the perfect gas equation. The derivation of $A(M)$ for water [Eq. (2.9)] was carried out with no reference to the energy equation, because Eq. (2.5) was 
assumed to be independent of entropy. On the other hand, $\mathrm{Holl}^{3}$ and $\mathrm{Best}^{20}$ use the Rankine Hugoniot relation for the conservation of energy to write an over-determined system which they use to derive $A(M)$ for water. Cates ${ }^{18}$ has shown that this approach can lead to unnecessary error when calculating the Mach number from pressure. The present methodology avoids this difficulty.

\section{CONCLUSIONS}

The finite-difference method for geometrical shock dynamics developed in this work provides an efficient and inexpensive tool for approximately solving problems of shock focusing where strong shock-shock discontinuities occur. The physical mechanisms which are influenced by aperture angle and initial Mach number are properly represented by geometrical shock dynamics. The numerical results exhibit the qualitative behavior of strong and moderate-strength shock focusing observed in experiments. A comparison with experimental measurements of the location at which maximum shock pressure occurs at a focus shows that shock dynamics gives accurate results, within the accuracy of the experiments, for strong and moderate shock waves. For weak shock waves, when the computations show a knee on the pressure profile, the agreement is equally good if the location of the knee is taken to be the focal point. However, owing to under-prediction of the speed of diffracted waves from the corner by shock dynamics, the pressure at the focus is overpredicted by approximately $60 \%$, and the strength of the shock-shock moving out from the centerline is correspondingly too large. Agreement degrades with decreasing shock strength, as is well known for shock dynamics. Furthermore, even for weak shock waves, far downstream from the focus the shock-shock always moves away from the axis of symmetry, and the reflection becomes irregular, in disagreement with experiment. This may simply be another example of qualitative but not quantitative agreement with experiment, or it may be a fundamental failure of shock dynamics theory. It remains a subject for future research. We conclude that shock dynamics calculations are useful for obtaining quick qualitative estimates of the geometrical configuration of complex weak-shock interactions, but that the quantitative values returned are of limited accuracy for moderate-strength shocks $\left(M_{0} \sim 1.2\right)$ and the accuracy worsens as the shock strength decreases.

\section{ACKNOWLEDGMENTS}

This work was supported by the NIDDK, National Institutes of Health under Grant No. P01 DK43881-01A3 and, in part, by a National Science Foundation Fellowship.

${ }^{1}$ B. Sturtevant and V. A. Kulkarny, "The focusing of weak shock waves," J. Fluid Mech. 73, 651 (1976).

${ }^{2}$ V. A. Kulkarny, "An experimental investigation of focusing of weak shock waves in air,' Ph.D. thesis, Graduate Aeronautical Laboratories, California Institute of Technology, Pasadena, California, 1975.

${ }^{3}$ R. Holl, "Wellenfokussierung in fluiden,' Dissertation, RWTH, Aachen, 1982

${ }^{4}$ M. Müller, "Experimental investigations on focusing of weak spherical shock waves in water by shallow ellipsoidal reflectors," Acustica 64, 85 (1987).

${ }^{5}$ M. Müller, "Die Fokussierung von wasserstobwellen zur lithotripsie durch verschiedene ellipsoidreflektoren,'” Biomed. Tech. 34, 62 (1989).

${ }^{6} \mathrm{G}$. B. Whitham, "A new approach to problems of shock dynamics. Part I. Two-dimensional problems,' J. Fluid Mech. 2, 145 (1957).

${ }^{7} \mathrm{G}$. B. Whitham, "A new approach to problems of shock dynamics. Part 2. Three-dimensional problems,', J. Fluid Mech. 5, 369 (1959).

${ }^{8}$ W. D. Henshaw, N. F. Smyth, and D. W. Schwendeman, "Numerical shock propagation using geometrical shock dynamics,' J. Fluid Mech. 171, 519 (1986).

${ }^{9}$ E. A. Zabolotskaya and R. V. Khokhlov, "Convergent and divergent sound beams in nonlinear media,' Sov. Phys. Acoust. 16, 39 (1970).

${ }^{10}$ Y. -S. Lee and M. F. Hamilton, "Time-domain modeling of pulsed finiteamplitude sound beams," J. Acoust. Soc. Am. 97, 906 (1995).

${ }^{11}$ M. Brio and J. K. Hunter, "'Mach reflection for the two-dimensional Burgers equation," Physica D 20, 194 (1992).

${ }^{12}$ A. E. Bryson and R. W. F. Gross, "Diffraction of strong shocks by cones, cylinders, and spheres,' J. Fluid Mech. 10, 1 (1961).

${ }^{13}$ C. J. Catherasoo and B. Sturtevant, "Shock dynamics in non-uniform media,' J. Fluid Mech. 127, 539 (1983).

${ }^{14}$ D. W. Schwendeman, "A new numerical method for shock wave propagation based on geometrical shock dynamics,' Proc. R. Soc. London Ser. A 441, 331 (1993).

${ }^{15}$ L. F. Henderson, "On the Whitham theory of shock-wave diffraction at concave corners,', J. Fluid Mech. 99, 801 (1980).

${ }^{16} \mathrm{~J}$. Kirkwood and H. Bethe, "Progress report on the pressure wave produced by an underwater explosion,', OSRD Report 588, 1942.

${ }^{17}$ V. Shankar and S.Osher, "An efficient, full-potential implicit method based on characteristics for supersonic flows," AIAA J. 21, 1262 (1983).

${ }^{18} \mathrm{~J}$. E. Cates, "Studies of shock wave focusing using geometrical shock dynamics,' Ph.D. thesis, California Institute of Technology, Pasadena, CA, 1996.

${ }^{19}$ J. -F. Haas and B. Sturtevant, "Interaction of weak shock waves with cylindrical and spherical gas inhomogeneities,' J. Fluid Mech. 181, 41 (1987).

${ }^{20}$ J. Best, "A generalization of the theory of geometrical shock dynamics,", Shock Waves 1, 251 (1991). 